MAGNETOHYDRODYNAMICS Vol. 54 (2018), No. 1-2, pp. 55-59

DOI: $10.22364 / \mathrm{mhd} .54 .1-2.10$

\title{
FEATURES OF ELECTRICAL PROPERTIES IN A STRUCTURED THIN MAGNETIC FLUID LAYER
}

\author{
V.M. Kozhevnikov, Yu.A.Larionov, I.Yu. Chuenkova \\ GAOWO North-Caucasus Federal University, Stavropol, Russian Federation \\ e-Mail:kvm@stv.runnet.ru
}

Investigations of the properties and application possibilities of magnetic fluids (MF) are an extremely dynamic research field nowadays. Many researchers, working in the field of MF physics and application, studied unique properties of magnetic fluids. Different physical phenomena determined by the MF application have been investigated. The growing interest caused by the use of magnetic fluids in instrumentation is determined by the MF interaction with electric and magnetic fields [1]. Studying a certain property of MF makes it impossible to eliminate the effect of more or fewer factors. On the one hand, it has some advantages since it allows to judge about the phenomenon from different points of view and, on the other hand, causes some difficulties when comparing results. As to the electrical properties (electrical conductivity, dielectric permittivity), it should be taken into account that they can be characterized by different methods using samples with different structures and produced by various methods. MFs have a more complex and diverse structure than most colloidal systems, because their structure is affected both by electric and magnetic fields.

Introduction. In this work, we consider these phenomena, starting with the effect of $\mathrm{AC}$ and $\mathrm{DC}$ electric fields on the MF layer. In the AC field, polarization appears, and if the frequency $\omega$ is large enough, the orientation of the dipoles inevitably lags after the applied field. It can be illustrated by the lag of the electrical displacement $D$ by an angle $\delta$. The vectors $\mathbf{E}$ and $\mathbf{D}$ are related by the complex dielectric permittivity $\varepsilon=\varepsilon^{\prime}-j \varepsilon^{\prime \prime}$ and $\operatorname{tg} \delta=\varepsilon^{\prime \prime} / \varepsilon^{\prime}$. The frequency dependences of $\varepsilon^{\prime}$ and $\varepsilon^{\prime \prime}$ show the adequacy of the Debye model to a real system. Maxwell (1892) and Wagner (1914) took into account the dielectric inhomogeneity and developed an approximate model of an insulator with conductive impurities [2]. If the fluid contains electrically conducting impurities, the so-called electrode polarization appears in it. The dielectric permittivity at low frequencies increases in samples with a high electrical conductivity. Johnson and Cole [2] defined the apparent dielectric permittivity as

$$
\varepsilon_{\text {sim }}=\varepsilon^{\prime}+\frac{\sigma C_{0}}{\omega^{2} \varepsilon_{0}^{2} C_{\mathrm{e}}},
$$

where $\varepsilon^{\prime}$ and $\sigma$ are the frequency-independent dielectric permittivity and the electrical conductivity of material, respectively, $C_{0}$ is the geometric capacity, $C_{\mathrm{e}}$ is the sample capacity. This formula, describing the properties of many fluids, allows correcting the electrode effects in the low-frequency range.

1. Experiment. The electrical properties of kerosene-based MFs were investigated by known methods. The electrical conductivity was determined by measuring the RMS values of the current, voltage and the phase angle between them. The dielectric permittivity was determined by the bridge method from the capacitance ratio of an empty cell and a cell with an MF layer. Electromagnets and Helmholtz coils created a magnetic field. The distance between the electrodes in a plane-parallel glass cell with an electrically conducting $\mathrm{In}_{2} \mathrm{O}_{3} \mathrm{SnO}_{2}$ cover was controlled by a PTFE gasket. The temperature regime was maintained by a thermostat. All samples were produced by diluting the original concentrated solution. 


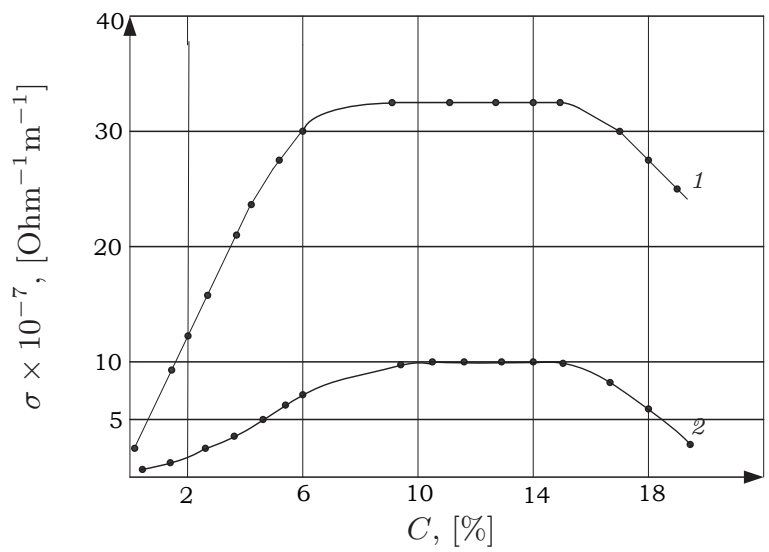

Fig. 1. The MF layer electrical conductivity vs. the concentration at a distance between the electrodes: $1-2 \mathrm{~mm}, 2-45 \mu \mathrm{m}$.

2. Results and discussion. The extreme character of the concentration dependence of the MF electrical conductivity obtained under laboratory conditions in Stavropol and in Minsk characterizes the MF electrical properties [3, 4]. The electrical conductivity of the MF layer at a micron distance between electrodes is reduced by a factor of 4 if compare with the electrical conductivity at a $2-\mathrm{mm}$ distance (Fig. 1) [4].

This feature served as the beginning of the studies of the MF thin layer electrical properties. The magnetic field significantly affected the electrical conductivity in the MF layer with a concentration of the solid phase corresponding to a maximum electrical conductivity and depended on the mutual orientation of the $\mathbf{E}$ and $\mathbf{H}$ vectors (Fig. 2). The effect of the magnetic field can be explained by the different ratio between the conductivity components of the magnetic particle mobility and the formation of conducting structures. If the fluid viscosity increases, the mobility of magnetite particles in the structures decreases. These two factors can decrease the total conductivity. The structures formed in the magnetic field can damage the particle surfactant protective layer and increase the electrical conductivity.

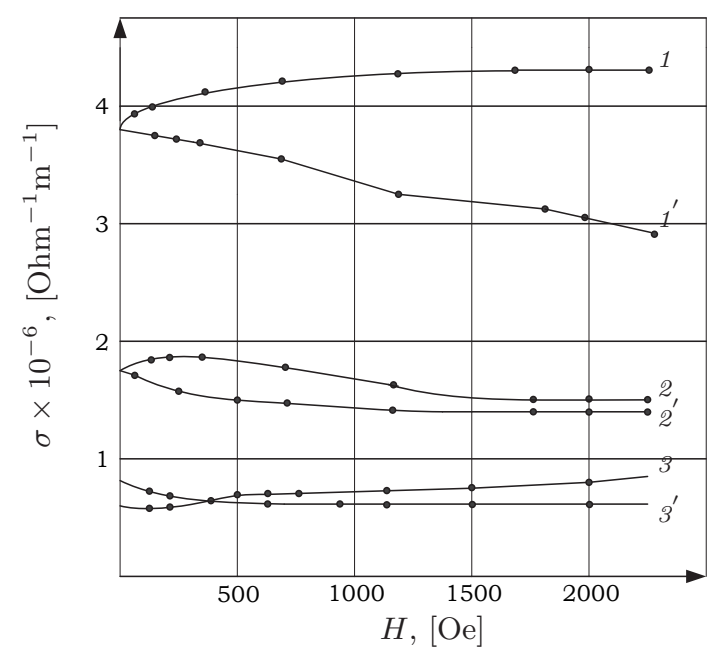

Fig. 2. The electrical conductivity of a magnetic fluid with different magnetite concentrations vs. the magnetic field: 1 and 1': $10.2 \%$, respectively, for $\mathbf{E} \| \mathbf{H}$ and $\mathbf{E} \perp \mathbf{H}$; 2 and 2': $2.49 \%$ for $\mathbf{E} \| \mathbf{H}$ and $\mathbf{E} \perp \mathbf{H}$; 3 and 3': $24.8 \%$ for $\mathbf{E} \| \mathbf{H}$ and $\mathbf{E} \perp \mathbf{H}$. 
Features of electrical properties in a structured thin magnetic fluid layer

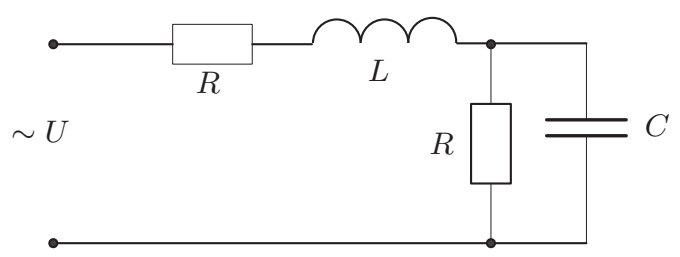

Fig. 3. An electrical model of the MF layer in an electric field.

As a result, the dependence of the MF layer electrical conductivity on the magnetic field is barely reproduced (Fig. 2) in time. If $\mathbf{E} \| \mathbf{H}$, the "conduction chains" are located perpendicular to the electrodes, and if $\mathbf{E} \perp \mathbf{H}$, the aggregates are located parallel to the electrodes and do not form "conduction chains", i.e. the particles mobility decreases. As a result, the electrical conductivity decreases, too. It is suggested that the magnetic field also affects the near-electrode areas, i.e. the electrode polarization.

The extreme character of the dependence of the capacitance change $\Delta \mathrm{C}$ on the polarizing voltage, as well as the negative real part of the complex dielectric permittivity at low frequencies allow to suggest that the electric field forms some crystal state in a thin MF layer similar to liquid ferroelectrics. So it is important to select a thin layer model in an electric field. This model must consider the current-voltage (CVC) and ampere-time (ATC) characteristics [7]. The ATC give current oscillations under the positive rectangular pulse. All known models consist of active-capacitive elements which exclude current oscillations. The oscillatory process can occur only in the RLC circuit. The intensive structural changes which form additional magnetic circuits can result in an oscillatory process and require an internal inductance $L$ to be introduced to the electrical model. The coefficient between the magnetic flux $\Phi$ and the current increases by the $n$-factor if the initial circuit is split into $N$ circuits. An estimation of the internal inductance due to the labyrinth and ring structures yields $L \cong 10^{-3} \mathrm{H}$. A feasible model is shown in Fig. 3.

In the general case, the total current through the MF layer is [8]

$$
J=U k^{-1}\left[\sigma_{o}+\varepsilon \omega \cos \varphi(\operatorname{tg} \varphi+j)\right]
$$

where $\sigma_{0}$ is the fluid electrical conductivity; $\varepsilon$ is the dielectric permittivity; $k=$ $\sigma_{0} / \sigma_{\text {ef }} ; \sigma_{\text {ef }}$ is the conductivity of the fluid layer; $\omega=2 \pi f$, where $f$ is the frequency; $\varphi$ stands for the angle of dielectric losses due to the fluid molecule polarization in a sinusoidal electric field. The angle of dielectric losses does not consider the losses caused by the free charged particle motion (conduction current). The value of $\operatorname{tg} \varphi$ does not include $\sigma$ o, i.e. $\operatorname{tg} \varphi=\varepsilon " / \varepsilon$ '. For low frequencies when $\operatorname{tg} \varphi \ll 1$, expression (1) yields

$$
J=U k^{-1}\left[\sigma_{o}+\varepsilon \omega \operatorname{tg} \varphi+j \varepsilon \omega\right] .
$$

According to Eq. (1) for the solutions in the AC electric field, the active part of the current consists of two components. The component $\varepsilon \omega \cos \varphi \operatorname{tg} \varphi$ represents the active conductivity, which depends on the dielectric permittivity modulus, frequency and dielectric losses angle when the molecules and particles oscillate in the AC field. From Eq. (1), the tangent of dielectric losses is

$$
\operatorname{tg} \delta=\frac{\sigma_{o}+\varepsilon \omega \cos \varphi \operatorname{tg} \varphi}{\varepsilon \omega \cos \varphi}=\frac{\sigma_{o}}{\varepsilon \omega \cos \varphi}+\operatorname{tg} \varphi
$$




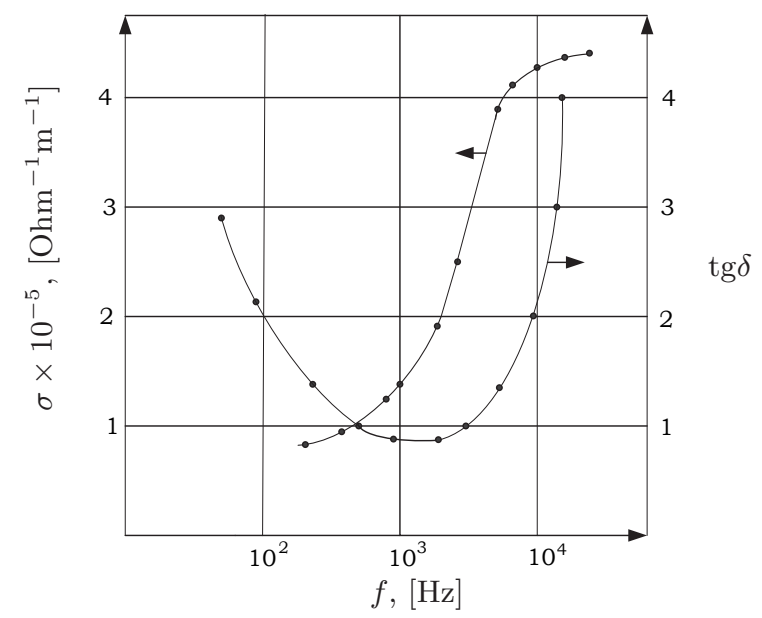

Fig. 4. Electrical conductivity and angle losses of the MF layer vs. frequency.

The decrease of $\operatorname{tg} \delta$ at low frequencies is attributed to the decrease of the first component in Eq. (3), whereas the second component at these frequencies is still small (Fig. 4). The rotation rate of the molecules and particles at these frequencies is small and corresponds to relatively small energy dissipation. If the frequency increases, this causes a significantly much more energy to reorient the molecules. The first and second components in Eq. (3) for the active conductivity become comparable. The minimum value corresponds to frequencies at which the decrease in losses due to the motion of free ions ceases to be predominant over the growth of the losses associated with the reorientation of the molecules and particles. Both components depend on the temperature, but their temperature coefficients are opposite in sign.

3. Conclusions. The peculiarities of the MF electrical properties give evidence that

(i) the presence of aggregates determines the extreme nature of the concentration dependence and dependence of the electrical conductivity on the magnetic field;

(ii) the electric double layer at the electrode surface creates conditions for the decrease of the electrical conductivity in MF layers less than $100 \mu \mathrm{m}$;

(iii) maximum changes in thin layer capacitance $\Delta \mathrm{C}$ from the polarizing voltage are typical of ferroelectric material and allow to suggest that the electric field forms a structural state similar to that of ferroelectric materials;

(iv) the effect of rotating rings and periodic vortical structures should be considered in the electrical model of a thin MF layer as an internal inductance;

(v) the minimum of the $\operatorname{tg} \delta$ frequency dependence can be explained by the change of the ratio of the first and second components in Eq. (3).

\section{References}

[1] V.M. Kozhevnikov, Yu.A. Larionov, I.Yu. Chuenkova. Electromagneto-optical activity of magnetic fluid in instrumentary. In: Proc. of the 16 International Scientific Conference on Nanodispersed Magnetic Fluids, (Pless, 2014), pp. 204-209 (in Russian). 
Features of electrical properties in a structured thin magnetic fluid layer

[2] A.R. Blythe, D. Bloor. Electrical Properties of Polymers (Cambridge University Press, 2005).

[3] G.M. Gordeev, N.P. Matusiewich, S.P. Rdzlska, V.E. Fertman. Electric Properties of Magnetic Fluids. In: Physical Properties of Magnetic Fluids (Sverdlovsk, 1983), pp. 98-102 (in Russian).

[4] Yu.I. Dykansky, V.M. Kozhevnikov, V.V. Chekanov. Magnetic susceptibility and electrical conductivity of magnetic fluid with structural aggregates. In: Physical Properties of Magnetic Fluids (Sverdlovsk, 1983), pp. 28-33 (in Russian).

[5] V.M. Kozhevnikov, I.Yu. Chuenkova, M.I. Danilov, S.S. Yastrebov. Dynamics of self-organization processes in a thin layer of magnetic fluid in a constant electric field. Journal of Technical Physics, vol.. 79 (2006), no. 7, pp. $129-131$.

[6] V.M. Kozhevnikov, I.Yu. Chuenkova., M.I. Danilov, S.S. Yastrebov. Voltage self-oscillations and dynamics of phase separation in a thin layer of weakly conducting ferrofluid during periodic electrohydrodynamic flows. Journal of Technical Physics, vol.. 78 (2008), no. 2, pp. 51-57.

[7] YU.A. LaRIONOV. Kinetics of ordering in magnetic colloid in a near-electrode layer (PhD Thesis, Stavropol Technical University Press, Stavropol, 2002).

[8] S.V. Usikov. Electrometry Fluids (Chemistry Press, Leningrad, 1974) (in Russian).

Received 27.12.2017 